\title{
INTERAÇÃO GENÓTIPO-AMBIENTE PARA A PRODUÇÃO DE LEITE EM REBANHOS DA RAÇA HOLANDESA NO BRASIL. (I) MODELO DE TOURO
}

\author{
GENOTYPE-ENVIRONMENT INTERACTION ON MILK PRODUCTION \\ IN HOLSTEIN IN BRAZIL. (I) SIRE MODEL
}

\author{
Paulo Roberto Nogara Rorato ${ }^{1}$, Rui da Silva Verneque ${ }^{2}$, Mário Luiz Martinez ${ }^{2}$, \\ Gilka Barbosa Ferreira ${ }^{1}$ e José Valente ${ }^{2}$
}

RESUMO

Com o objetivo de avaliar o efeito da interação genótipo-ambiente sobre o desempenho produtivo de vacas da raça Holandesa no Brasil, foram estudados os registros de produção total de leite à primeira lactação de 14.418 vacas filhas de 324 touros e distribuídas em 181 rebanhos em diferentes estados, no período de 1981 a 1991. Os dados foram estratificados de acordo com a produção média de leite do rebanho, em nível baixo $(B)$, médio $(M)$ e alto (A). Os componentes de (co)variância foram estimados utilizando-se o método da máxima verossimilhança restrita e dois modelos de touro. Os componentes de variância de touro variaram de 116.879 a 274.871 e foram maiores nos níveis mais altos; os residuais variaram de 1.691 .879 a 1.956.025, crescendo com o nível de produção dos rebanhos e os da interação variaram de 66.854 a 149.972, tendo o maior valor ocorrido nos níveis extremos de produção. Os coeficientes de herdabilidade variaram de 0,22 a 0,49 e os de correlação genética foram $0,22,0,46$ e 0,69, entre os níveis $B$ e A, B e M e M e A, respectivamente.

Palavras-chave: interação genótipo-ambiente, produção de leite, máxima verossimilhança restrita.

\section{SUMMARY}

Records on 14.418 first lactations of Holstein cows sired by 324 bulls distributed in 181 herds in different States from 1981 to 1991, were used to study the effect of genotypeenvironment interaction on milk production. The data were distributed in three levels (low-B, medium- $M$, and high-A) according to the average of the herd milk production. (Co)variances components were estimated by REML using two sire models. The variance components of sire ranged from 116,879 to 274,871 were larger at the higher levels, the residuals ranged from 1,691,879 to 1,956,025, increasing with the production level of the herds and the interaction ranged from
66,854 to 149,972 with the highest value when the daughters performed at the extreme levels. The heritabilities ranged from 0.22 to 0.49 and the genetic correlations were 0.22, 0.46, and 0.69 , respectivelly, among the levels low and high, low and medium, and medium and high.

Key words: genotype by environment interaction, milk production, restricted maximum likelihood.

\section{INTRODUÇÃO}

Com o objetivo de melhorar geneticamente os rebanhos leiteiros é prática comum o uso de sêmen importado. Entretanto, freqüentemente, verifica-se que a produção média de um grupo de filhas de determinado reprodutor tem sido inferior em nosso meio quando comparada aos resultados obtidos no país de origem do reprodutor.

$\mathrm{Na}$ tentativa de elucidar a questão da importância da interação genótipo-ambiente em gado leiteiro, inúmeros trabalhos de pesquisa têm sido realizados, estimando a correlação genética entre o desempenho das filhas de um mesmo reprodutor em diferentes condições de ambiente. Os valores encontrados para as estimativas do coeficiente de correlação genética, nos trabalhos mais recentes, foram: 0,26 , entre a ordem dos valores genéticos de touros da raça Holandesa no México e na Colômbia (ABUBAKAR et $\boldsymbol{a l}$., 1987); 0,85, entre os valores genéticos dos touros em diferentes níveis de produção nos Estados Unidos da América (DE VEER \&

\footnotetext{
${ }^{1}$ Professor Adjunto, Douutor, Departamento de Zootecnia, Centro de Ciências Rurais, Universidade Federal de Santa Maria, 97105-900, Santa Maria, RS. E-mail: rorato@ccr.ufsm.br, Autor para correspondência.

${ }^{2}$ Pesquisador, Doutor, CNPGL-EMBRAPA, 36038-330, Juiz de Fora, MG.
} 
VAN VLECK, 1987); 0,81, entre o desempenho das filhas de um mesmo reprodutor na Espanha e nos Estados Unidos da América-EUA (CARABAÑO $\boldsymbol{e t}$ al., 1989); superiores a 0,90, entre o desempenho de vacas da raça Holandesa, filhas de um mesmo reprodutor, em diferentes regiões dos EUA (CARABAÑO et al., 1990); altas, entre o desempenho de vacas da raça Holandesa nos EUA, Colômbia e México (STANTON et al., 1991b); 0,25 e 0,36, entre o desempenho de vacas da raça Holandesa, filhas de um mesmo reprodutor, em diferentes regiões do Estado do Paraná (RORATO et al., 1994); 0,33, entre os níveis de produção baixo e alto; 0,69, entre os níveis baixo e médio e 0,98 , entre os níveis médio e alto, em rebanhos da raça Holandesa no Brasil (RORATO et al., 1996).

O objetivo deste trabalho foi avaliar a ocorrência da interação genótipo-ambiente sobre a produção total de leite na raça Holandesa através da estimativa do coeficiente de herdabilidade em três níveis de produção e do coeficiente de correlação genética entre as produções das filhas de um mesmo reprodutor nos níveis (ambientes) diferentes, utilizando-se a fórmula de YAMADA (1962).

\section{MATERIAL E MÉTODOS}

$\mathrm{O}$ arquivo de dados estudado constituiu-se dos registros de produção total de leite à primeira lactação de 14.418 vacas da raça Holandesa filhas de 324 touros e distribuídas em 181 rebanhos em diferentes estados do Brasil, no período de 1981 a 1991. Os dados foram extraídos do Arquivo Zootécnico Nacional-Gado de Leite, localizado no CNPGL/ EMBRAPA. Sob a suposição de que nos rebanhos de produção média mais baixas, o manejo, em geral, é deficitário, tornando-se mais eficiente nos rebanhos de médias mais elevadas, o ambiente foi diferenciado de forma indireta, pela média de produção do rebanho. Os dados foram estratificados de acordo com a produção média de leite do rebanho, em nível baixo (B) para as produções por lactação, ajustadas à idade adulta, abaixo de $5.500 \mathrm{~kg}$, médio (M) de 5.001 a $6.500 \mathrm{~kg}$ e alto (A) acima de $6.500 \mathrm{~kg}$. Posteriormente, foram agrupados em três conjuntos: BM, BA e MA, reunindo as produções das filhas dos touros com ocorrências, respectivamente, nos níveis $\mathrm{B}$ e $\mathrm{M}$, $\mathrm{B}$ e A e M e A. Os componentes de (co)variância foram estimados, utilizando-se o método da máxima verossimilhança restrita (REML) e dois modelos de touro. No primeiro (I), as produções totais de leite foram estudadas em função dos efeitos fixos de ano-época, nível de produção e rebanho: nível de produção e os aleatórios de touro, da interação touro-nível de produção e do resíduo. O segundo modelo (II) incluiu os efeitos fixos de ano-época e rebanho e os aleatórios de touro e resíduo. Cada um dos conjuntos de dados anteriormente descritos foi analisado pelo modelo I , com o objetivo de estimar os componentes de variância de touro e da interação touro-nível e pelo modelo II para cada nível dentro do conjunto, com o objetivo de estimar os desvios-padrões genotípicos (efeito de touro) nos ambientes distintos. Estes componentes foram utilizados para estimar os coeficientes de herdabilidade nos diferentes níveis e na fórmula de YAMADA (1962), abaixo descrita, para estimar os coeficientes de correlação genética, medida indireta da interação genótipo-ambiente: $r_{g}=\sigma_{a}^{2}-1 / k \sigma_{I}^{2} / \sigma_{a}^{2}+1 / k \sigma_{I}^{2}-1 / 2\left(\sigma_{a 1}-\sigma_{a 2}\right)^{2} ;$ em que $r_{g}=$ estimador do coeficiente de correlação genética entre os valores genéticos; $\sigma_{\mathrm{a}}^{2}=$ estimador do componente de variância entre touros; $\sigma_{I}^{2}=$ estimador do componente de variância da interação touro-ambiente; $\mathrm{k}=$ número de ambientes; $\sigma_{\mathrm{a} 1}, \sigma_{\mathrm{a} 2}=$ estimadores dos desvios-padrões genotípicos para os dois ambientes. O programa computacional utilizado foi o MTDFREML desenvolvido por BOLDMAN et al. (1995).

\section{RESULTADOS E DISCUSSÃO}

A distribuição dos dados pelos níveis está apresentada na tabela 1 , onde se pode observar uma maior concentração de informações nos níveis médio e alto com, aproximadamente, o dobro de observações em relação ao nível baixo. Observa-se na tabela 1 que com um número de touros cerca de $7 \%$ menor e de animais cerca de 50\% menor que os outros, o nível baixo apresentou o menor número médio de filhas por touro, provavelmente, devido a uma maior rotatividade na utilização dos reproduto-

Tabela 1 - Distribuição dos touros, das lactações, do número de filhas por touro, das produções médias de leite e dos coeficientes de variação através dos níveis.

\begin{tabular}{lccccc}
\hline Níveis & Touros & Filhas & Filhas/ touro & Leite & $\mathrm{CV}^{2}$ \\
& & & & & \\
\hline & & & & & \\
Baixo - B & 278 & 2.768 & 10 & 4.911 & 29 \\
Médio - M & 298 & 5.850 & 20 & 6.046 & 24 \\
Alto - A & 301 & 5.800 & 19 & 7.070 & 22 \\
& & & & & \\
\hline & 324 & 14.418 & 45 & 6.240 & 27 \\
Total & & & & & \\
\hline
\end{tabular}

${ }^{1}$ Produção média de leite, em kg.

${ }^{2}$ Coeficiente de variação, em $\%$. 
res, sugerindo a ausência de qualquer critério seletivo. O coeficiente de variação de $29 \%$ para o nível baixo, maior do que aqueles observados para os níveis médio e alto, sugere ser o potencial produtivo das vacas deste nível bastante variável.

Na tabela 2, é apresentada a distribuição dos touros e das filhas nos diferentes conjuntos de dados e verifica-se um número maior de touros (cerca de $7 \%$, em relação aos conjuntos BA e BM) e de filhas (cerca de $28 \%$, em relação aos conjuntos BA e $\mathrm{BM})$ no conjunto MA. Com relação ao número de filhas por nível, dentro dos conjuntos, observa-se uma grande diferença, cerca de $48 \%$, nos conjuntos $\mathrm{BM}$ e BA, sugerindo que os touros, provavelmente, tenham sido usados mais intensivamente nos níveis mais altos do que nos mais baixos.

Os componentes de variância de touro estimados nos diferentes níveis de produção variaram de 116.879 a 274.871 (tabela 3) e foram maiores nos níveis mais altos, concordando com o relatado por DE VEER \& VAN VLECK (1987), os quais observaram um aumento na magnitude do componente de variância de reprodutor e do resíduo com o aumento do nível de produção dos rebanhos. Concordam também com STANTON et al. (1991a), os quais verificaram que, em dados da América Latina com médias de produção semelhantes às dos EUA, as variâncias de touro foram menores e com STANTON et al. (1991b) que relataram ter sido a resposta média das filhas à seleção dos touros, para a produção de leite, menor na América Latina em relação aos EUA, sugerindo que menor variância genética é expressa nos ambientes subtropical e tropical.

Os componentes de variância residuais (tabela 3) variaram de 1.691 .879 a 1.956 .025 , crescendo com o nível de produção dos rebanhos, sendo no nível $\mathrm{B}$ de 4 a $13 \%$, menor do que no nível A,

Tabela 2 - Distribuição dos touros e das filhas nos conjuntos de dados e nos níveis, dentro de conjunto.

\begin{tabular}{lcccc}
\hline Conjuntos & Touros & Filhas & $\begin{array}{l}\text { Níveis/ } \\
\text { Conjunto }\end{array}$ & $\begin{array}{l}\text { Filhas/ } \\
\text { Conjunto }\end{array}$ \\
\hline & & & & \\
BM & 262 & 8.344 & B & 2.264 \\
& & & M & 5.670 \\
BA & 259 & 8.255 & B & 2.666 \\
& & & A & 5.589 \\
MA & & & M & 5.755 \\
& 279 & 11.452 & A & 5.697 \\
\hline
\end{tabular}

Tabela 3 - Componentes de variância estimados para os efeitos de touro $\left(\sigma_{\mathrm{s}}^{2}\right)$, do resíduo $\left(\sigma_{\mathrm{e}}^{2}\right)$ e da interação touro-nível de produção $\left(\sigma^{2}\right)$ nos diferentes conjuntos de dados.

\begin{tabular}{lccc}
\hline Níveis & $\sigma^{2}{ }_{\mathrm{s}}$ & $\sigma_{\mathrm{e}}^{2}$ & $\sigma_{\mathrm{I}}^{2}$ \\
\hline & & & \\
$\mathrm{BM}$ & 125.006 & 1.825 .686 & 92.336 \\
$\mathrm{~B}$ & 203.397 & 1.691 .879 & - \\
M & 219.357 & 1.875 .288 & - \\
BA & 116.879 & 1.884 .179 & 149.972 \\
B & 218.871 & 1.697 .321 & - \\
A & 274.871 & 1.956 .025 & - \\
MA & 177.951 & 1.914 .030 & 66.854 \\
M & 209.706 & 1.874 .107 & - \\
A & 274.439 & 1.949 .783 & - \\
& & & \\
\hline
\end{tabular}

dentro dos conjuntos. Estes resultados estão de acordo com os de DE VEER \& VAN VLECK (1987) e com STANTON et al. (1991b), que observaram comportamento semelhante para a variância residual.

Os componentes de variância da interação touro-nível de produção (tabela 3) variaram de 66.854 a 149.972, tendo o maior valor ocorrido para produções nos níveis extremos BA e o menor valor ocorrido nos níveis MA.

Os coeficientes de herdabilidade estimados variaram de 0,22 a 0,49 (tabela 4), nos conjuntos de dados BM e BA, respectivamente. Os coeficientes de herdabilidade estimados foram maiores no nível de produção $\mathrm{B}$, o que está em desacordo com os resultados dos trabalhos realizados por ABUBAKAR $\boldsymbol{e t}$ al. (1987), DE VEER \& VAN VLECK (1987) e CARABAÑO et al. (1989), os quais verificaram que os coeficientes de herdabilidade eram maiores nos níveis mais altos. Entretanto, para o conjunto de dados MA, o coeficiente de herdabilidade estimado foi $22,5 \%$ maior para o nível de produção A, onde o ambiente mais favorável permitiu uma melhor expressão dos genótipos, concordando com os resultados observados pelos autores acima citados.

Os coeficientes de correlação genética estimados foram 0,46 e 0,22 entre os níveis baixo e médio e baixo e alto, respectivamente (tabela 4). A menor estimativa ocorreu no conjunto de dados BA, conseqüência da grande diferença no desempenho das filhas de um mesmo reprodutor nos níveis extremos, provavelmente por não terem as filhas tido a oportunidade de expressarem sua real capacidade de produção em conseqüência das limitações impostas pelo ambiente no nível mais baixo, caracterizando a 
Tabela 4 - Coeficientes de herdabilidade $\left(h^{2}\right)$ e de correlação genética $\left(r_{g}\right)$, de acordo com os diferentes conjuntos de dados.

\begin{tabular}{cccccccccc}
\hline Níveis & BM & B & M & BA & B & A & MA & M & A \\
\hline $\mathrm{h}^{2}$ & 0,25 & 0,43 & 0,42 & 0,22 & 0,46 & 0,40 & 0,22 & 0,40 & 0,49 \\
$\mathrm{r}_{\mathrm{g}}$ & 0,46 & - & - & 0,22 & - & - & 0,69 & - & -
\end{tabular}

expectativas de aumento de produção frustradas.

\section{AGRADECIMENTOS}

O autor agradece à Coordenadoria de Aperfeiçoamento de Pessoal de Ensino Superior CAPES e ao Conselho Nacional de Desenvolvimento Científico e Tecnológico - CNPq, pelo suporte financeiro e à Empresa Brasileira de Pesquisa Agropecuária - Centro Nacional de Pesquisa de Gado de Leite (EMBRAPA - CNPGL), pela cedência dos arquivos de registro de produção.

interação genótipo-ambiente. $\mathrm{O}$ efeito da interação genótipo-ambiente foi menor entre os níveis médio e alto, no conjunto de dados BM. Estes baixos valores podem também terem sido determinados, em parte, pelo pequeno número de observações no nível baixo. Estes resultados concordam com aqueles obtidos por ABUBAKAR et al. (1987), RORATO et al. (1994) e RORATO et al. (1996). Entretanto, discordam daqueles observados por DE VEER \& VAN VLECK (1987), CARABAÑO et al. (1989 e 1990) e STANTON et al. (1991b). O maior coeficiente de correlação genética estimado neste estudo, 0,69, sugere que mesmo entre os níveis médio e alto, com ambientes mais favoráveis, a resposta em termos produtivos das filhas de um mesmo touro foi diferente. Este valor é menor do que aqueles relatados por DE VEER \& VAN VLECK(1987) e por CARABAÑO et al. (1989 e 1990), provavelmente, em função de que, no material estudado, as diferenças de ambiente entre os níveis tenham sido maiores.

\section{CONCLUSÕES}

A variância de reprodutor cresce com o aumento do nível de produção do rebanho, sugerindo que quando o ambiente é favorável, as progênies conseguem expressar seu potencial genético, diferenciando-se umas das outras e permitindo uma melhor identificação dos reprodutores geneticamente superiores. O maior coeficiente de herdabilidade estimado, $27 \%$ maior do que o menor, pode levar a super ou subestimação da expectativa de produção das filhas nos diferentes níveis.

Os coeficientes de correlação genética estimados para os níveis BM e BA, 0,22 e 0,46, respectivamente, mostram o efeito da interação genótipo-ambiente de forma mais contundente e sugerem que os melhores reprodutores no nível mais baixo de manejo não o são, necessariamente, nos níveis médio e alto e vice-versa. Para o conjunto MA, o coeficiente de correlação genética estimado $(0,69)$ sugere um menor efeito da interação genótipo-ambiente.

Os resultados deste estudo indicam que ao selecionar o sêmen a ser utilizado no rebanho o produtor deve levar em consideração as condições de ambiente que terão durante sua vida produtiva os animais a serem gerados, sob pena de terem suas

\section{REFERÊNCIAS BIBLIOGRÁFICAS}

ABUBAKAR, B.Y., McDOWEL, R.E., VAN VLECK, L.D Interaction of genotype and environment for breeding efficiency and milk production of Holsteins in Mexico and Colombia. Trop Agric, Trinidad, v. 64, n. 1, p. 17-22, Jan, 1987.

BOLDMAN, K.G., KREISE, L.A., VAN VLECK, L.D., $\boldsymbol{e}$ t al. A Manual of MTDFREML. A Set of Programs To Obtain Estimates of Variances and Covariances (DRAFT). Lincoln: Departament of Animal Science/Agricultural Research Service, 1995. 114 p.

CARABAÑO, M.J., WADE, K.M., VAN VLECK, L.D. Genotype by environment interations for milk and fat production across regions of the United States. J Dairy Sci, Champaign, v. 73, n. 1, p. 173-180, Jan, 1990.

CARABAÑO, M.J., WIGGANS, G.R., ALENDA, R. Estimation of genetic parameters for milk and fat yields of dairy cattle in Spain and the United States. J Dairy Sci, Champaign, v. 72, n. 11, p. 3013-3022, Nov, 1989.

DE VEER, J.C., VAN VLECK, L.D. Genetic parameters for first lactation milk yields at three levels of her production. J Dairy Sci, Champaign, v. 70, n. 7, p. 1434-1441, Jul, 1987.

RORATO, P.R.N., LÔBO, R.B., FILHO, R.M., et al. Efeito da interação genótipo-ambiente sobre a produção de leite da raça Holandesa no Estado do Paraná. Rev Soc Bras Zoot, Viçosa, v. 23, n. 5, p. 859-869, Set/Out, 1994.

RORATO, P.R.N., MARTINEZ, M.L., VERNEQUE R. S. $\boldsymbol{e}$ t al. Estudo do efeito da interação genótipo-ambiente sobre a produção de leite da raça Holandesa no Brasil. In: REUNIÃO ANUAL DA SOCIEDADE BRASILEIRA DE ZOOTECNIA, 33, 1996, Forataleza, Anais... Fortaleza: SBZ, 1996. 677 p. p. $1-2$.

STANTON, T.L., BLAKE, R.W., QUAAS, R.L., et al. Response to selection of United States Holstein sires in Latin America. J Dairy Sci , Champaign, v. 74, n. 2 p. 651-664, Fev, 1991a.

STANTON, T.L., BLAKE, R.W., VAN VLECK, L.D. $\boldsymbol{e} \boldsymbol{t} \boldsymbol{a l}$. Genotype by environment interation for Holstein milk yield in Colombia, Mexico, and Porto Rico. J Dairy Sci, Champaign, v. 74 , n. 5, p. 1700-1714, May, 1991 b.

YAMADA, Y. Genotype by environment interaction and genetic correlation of the same trait under different environment. Jap Jour Genet, v. 37, p. 498-509, Jun. 1962. 